\title{
SYMBOLIC SOLUTION OF CERTAIN PROBLEMS IN PERMUTATIONS
}

\author{
IRVING KAPLANSKY
}

1. Introduction. It is our purpose in this paper to show how algebraic symbolism can be applied to the problem of enumerating certain types of restricted permutations. The method rests on a symbolic interpretation of the method of inclusion and exclusion, or rather its probability analogue, the formula of Poincaré; the use of the terminology of probability is a matter of convenience only, the considerations being purely combinatorial. The problems considered are of two kinds: those where the restriction of position of elements is absolute ( $\$ 3$, "card-matching" problems), and those where elements are restricted relative to others $(\$ \S 5,6)$. In $\S 4$ approximations to the symbolic formulas are obtained. Though these problems are rather special in character, the foundations of the method (\$2) are quite general and should find other applications.

2. Symbolic expressions. Consider $n$ events $A_{1}, \cdots, A_{n}$ and let $p\left(A_{i_{1}} \cdots A_{i_{k}}\right)$ denote the probability of the joint occurrence of $A_{i_{1}} \cdots A_{i_{k}}$. The probability that none of $A_{1}, \cdots, A_{n}$ occurs, which we shall denote by $P_{0}$, is given by Poincaré's formula:

$$
P_{0}=1-\sum p\left(A_{i}\right)+\sum p\left(A_{i} A_{j}\right) \cdots .
$$

In the case of complete symmetry, that is, where each $P\left(A_{i_{1}} \cdots A_{i_{k}}\right)$ is a function $\phi_{k}$ of $k$ alone,

$$
P_{0}=1-n \phi_{1}+{ }_{n} C_{2} \phi_{2}-\cdots \text {. }
$$

By using the displacement operator $E$ defined by $E^{k} \phi_{0}=\phi_{k}$, we may write more compactly $P_{0}=(1-E)^{n} \phi_{0}$.

In the cases which we shall consider, however, this complete symmetry will be lacking and instead we shall have the following partial substitute, which we shall call quasi-symmetry: $p\left(A_{i_{1}} \cdots A_{i_{k}}\right)$ is either equal to zero or to a function $\phi_{k}$ of $k$ alone. To evaluate $P_{0}$ for such cases a symbolic device of Broderick [2] ${ }^{1}$ is helpful; we may write (1) in the form

$$
P_{0}=\left(1-p A_{1}\right)\left(1-p A_{2}\right) \cdots\left(1-p A_{n}\right),
$$

where the multiplication is symbolic in the sense that $p\left(A_{i}\right) p\left(A_{j}\right) \ldots$

Received by the editors March 9, 1944.

1 Numbers in brackets refer to the Bibliography at the end of the paper. 
is interpreted as $p\left(A_{i} A_{j} \cdots\right) .^{2}$ The following rule can then be stated.

To evaluate (2) in the case of quasi-symmetry, first suppress all products that vanish; from the remaining expression form a polynomial $f(E)$ by replacing each term $p\left(A_{i}\right)$ by $E$. Then $P_{0}$ will equal $f(E) \phi_{0}$.

The advantage of this point of view lies in the fact that in the computation of $f(E)$ we may resort to all the devices of formal algebra. A second advantage, perhaps more important, was pointed out by Fréchet [3]. Once $f(E)$ has been found, we have simultaneously solved the problem of finding $P_{r}$, the probability that exactly $r$ of the events $A_{1}, \cdots, A_{n}$ occur. For Poincaré's formula gives

$$
P_{r}=\sum_{k=r}^{n}(-1)^{k-r_{h} C_{k-r}} \sum p\left(A_{i_{1}} \cdots A_{i_{k}}\right)
$$

which can be rewritten

$$
P_{r}=\sum_{k=0}^{n}(-1)^{r_{k}} C_{r} \sum(-1)^{k} p\left(A_{i_{1}} \cdots A_{i_{k}}\right) .
$$

Comparison of (1) and (3) shows that we may use the same polynomial $f(E)$ to evaluate $P_{r}$ provided we replace $\phi_{k}$ by $(-1)^{r}{ }_{k} C_{r} \phi_{k}$; that is,

$$
P_{r}=f(E) \psi_{0} \quad \text { where } \quad \psi_{k}=(-1)^{r}{ }_{k} C_{r} \phi_{k} .
$$

Moreover, since

$$
{ }_{k} C_{0}-{ }_{k} C_{1}+{ }_{k} C_{2}-\cdots+(-1)^{r_{k}} C_{r}=(-1)^{r}{ }_{b-1} C_{r},
$$

the use of $(-1)^{r}{ }_{k-1} C_{r} \phi_{k}$ will give the probability that at most $r$ of the $n$ events happen.

3. Card-matching problems. ${ }^{3}$ A general version of the so-called "card-matching" problem can be formulated as follows : in a permutation of $1, \cdots, n$ what is the probability that the integer $i$ is not in the $j$ th place, also $k$ is not $l$ th, and so on? More generally we can ask for the probability that these conditions are violated precisely $r$ times. For a convenient notation let $p_{i j}$ denote the probability that $i$ is $j$ th; then we are to evaluate a product of the form

$$
\left(1-p_{i j}\right)\left(1-p_{b l}\right) \cdots \text {. }
$$

We must first observe that the condition of quasi-symmetry is fulfilled; in fact the (symbolic) product of $k$ of the p's is either 0 or

2 We refrain from introducing a special notation for this symbolic multiplication, as it will always be clear from the context when it is intended.

${ }^{3}$ Cf. [1] for an extensive bibliography. 
$(n-k) ! / n !$. In accordance with our previous notation we shall therefore write

$$
\phi_{k}=(n-k) ! / n !, \quad \psi_{k}=(-1)^{r_{k}} C_{r}(n-k) ! / n !
$$

in the remainder of the paper. The question of determining when a product of $p_{i j}$ 's vanishes is readily answered; $p_{i j} p_{k l}=0$ if $i=k$ or $j=l$ and a product of several $p$ 's is 0 if and only if it contains such a pair. This observation makes it comparatively easy to compute $f(E)$ in some famous problems of the card-matching type.

In each of the problems that follows, the solution is provided by finding the polynomial $f(E)$ defined in $\$ 2$; the probability that a permutation violates the conditions $r$ times is $f(E) \psi_{0}$, with $\psi_{k}=E^{k} \psi_{0}$ given in (4).

(i) The classical "problème des rencontres" requires merely that $i$ shall not be $i$ th. We have to compute $\left(1-p_{11}\right) \cdots\left(1-p_{n n}\right)$. No products vanish so this is $(1-E)^{n}$.

(ii) In a widely known generalization of the preceding problem, the $n$ integers are divided into $n / a$ subsets of $a$ each, and every integer is forbidden to appear in each of the $a$ places originally occupied by the members of its subset. Thus, with $a=2$, we can suppose the elements so numbered that the subsets are $(1,2),(3,4), \cdots$, and the conditions read: neither 1 nor 2 is 1 st or 2 nd, neither 3 nor 4 is 3 rd or 4 th, and so on. We find $n / 2$ groups of 4 factors, the first of which is

$$
\begin{aligned}
\left(1-p_{11}\right)\left(1-p_{12}\right) & \left(1-p_{21}\right)\left(1-p_{22}\right) \\
= & 1-p_{11}-p_{12}-p_{21}-p_{22}+p_{11} p_{22}+p_{12} p_{21} .
\end{aligned}
$$

All vanishing products having now been eliminated, we have the answer $\left(1-4 E+2 E^{2}\right)^{n / 2}$. For the usual deck of 52 cards a similar calculation yields

$$
\left(1-16 E+72 E^{2}-96 E^{3}+24 E^{4}\right)^{13},
$$

and for the $5 \times 5$ deck used in telepathic experiments:

$$
\left(1-25 E+200 E^{2}-600 E^{3}+600 E^{4}-120 E^{5}\right)^{5} \text {. }
$$

We can generalize somewhat further by supposing that each of the $a$ members of a subset are forbidden to appear in $b$, rather than $a$, places. We have then to compute

$$
\begin{aligned}
F(a, b) & =\prod_{\left(1-p_{i j}\right) \quad(i=1, \cdots, a ; j=1, \cdots, b)} \\
& =\sum_{t=0}^{a}{ }_{a} C_{t} C_{b} t !(-E)^{t},
\end{aligned}
$$

as follows from a simple combinatorial argument. We can even sup- 
pose that the subsets are of different sizes $a_{1}, a_{2}, \ldots$ with the members forbidden $b_{1}, b_{2}, \cdots$ places respectively; this generalization leads to the polynomial $F\left(a_{1}, b_{1}\right) F\left(a_{2}, b_{2}\right) \ldots$. We remark again that by applying this operator to $\psi_{0}, \psi$ defined as in (4), we obtain the probability that a permutation will violate the stated conditions precisely $r$ times. This result was published without proof in [4].

(iii) In the "problème des ménages" the conditions read: 1 shall not be 1 st or 2 nd, 2 shall not be 2 nd or 3 rd, $\cdots, n$ not $n$th or 1 st. The product

$$
\left(1-p_{11}\right)\left(1-p_{12}\right) \cdots\left(1-p_{n n}\right)\left(1-p_{n 1}\right)
$$

was evaluated in [5] and found to be

$$
U_{n}=\sum_{k=0}^{n}{ }_{2 n-k} C_{k} 2 n(-E)^{k} /(2 n-k) .
$$

But we are able further to solve Lucas' more difficult problem [6, p. 491] of finding the number of permutations "discordant" with any two given ones. ${ }^{4}$ (The problème des ménages is the case where the given permutations are $1,2, \cdots, n$ and $2,3, \cdots, n, 1$.) The solution is as follows : considering one of the given permutations to be a permutation of the other, represent it as a product of disjoint cycles of length $a, b, c, \cdots$. Then the polynomial giving the probability of a permutation discordant with both is $U_{a} U_{b} U_{c} \cdots$, where $U_{n}$ is given by (6) for $n \neq 1$ and $U_{1}=1-E$.

(iv) As a final somewhat more intricate example, suppose $1, \cdots, n$ to be divided again into subsets of $a$ each, and let every integer be forbidden to appear in the places occupied by all the other members of its subset-its own original position is permitted. The answer, which we state without proof, is

$$
\left[F(a, a)+a E F(a-1, a-1)+{ }_{a} C_{2} E^{2} F(a-2, a-2)+\cdots+E^{a}\right]^{n / a} \text {, }
$$

$F$ being defined as in (5).

4. Asymptotic approximations. The symbolic formulas obtained in $\$ 3$ provide the exact answers to the problems in question and are readily calculated for small values of $n$. For large values of $n$ their exact evaluation is out of the question, but we can readily find approximations. The method is sufficiently well illustrated by a

${ }^{4}$ In an article submitted to the American Mathematical Monthly, John Riordan gives a detailed investigation of this solution, identifying it with one of Touchard [8], and applying it to the enumeration of $n$ by 3 Latin rectangles. 
consideration of the symbolic expression $(1-a E)^{2 n} \phi_{0}$, where $\phi_{k}$ is defined as in (4) and $z n$ is a positive integer not exceeding $n$.

$$
(1-a E)^{z n} \phi_{0}=\sum_{k=0}^{n}(-a)^{k}(z n)_{k} / k ! n_{k},
$$

where $n_{k,}=n(n-1) \cdots(n-k+1)$. Since

$$
(z n)_{k} / n_{k}=z^{k}-\left(k^{2}-k\right)\left(z^{k-1}-z^{k}\right) n^{-1} / 2+O\left(n^{-2}\right),
$$

it is natural to conclude from (7) and (8) that

$$
(1-a E)^{z n} \phi_{0}=e^{-a z}\left[1-a^{2}\left(z-z^{2}\right) n^{-1} / 2+O\left(n^{-2}\right)\right] .
$$

However, since the error term of (8) involves $k$, a more detailed investigation is necessary.

Let $m=\left[n^{1 / 8}\right]$. We split the sum (7) into two parts: $S_{1}$, the sum from $k=0$ to $m-1$, and $S_{2}$, the sum from $m$ to $n$. In all the applications $z \leqq 1$ and we shall treat only that case. Then $(z n)_{k} \leqq n_{k}$ and

$$
\left|S_{2}\right| \leqq \sum_{m}^{\infty}|a|^{k} / k !=O\left(m^{-6}\right)=O\left(n^{-2}\right)
$$

To estimate $S_{1}$ the following lemma is convenient.

LEMMA. Suppose $k \leqq m$ and let $R$ denote the sum of the absolute values of the terms in $n_{k}$ which are of degree not greater than $k-2$ in $n$. Then $R<k^{5} n^{k-2}$.

Proof. The term in $n^{i}$ is less than

$$
n^{i}(1+2+\cdots+k-1)^{k-i}<n^{i} k^{2 k-2 i} \text {. }
$$

Now $i \leqq k-2$ implies

$$
i+(2 k-2 i-4) / 3 \leqq k-2 .
$$

Hence $n^{i} k^{2 k-2 i} \leqq k^{4} n^{k-2}$. Since $R$ contains $k-1$ terms all less than $k^{4} n^{k-2}$, we have $R<k^{5} n^{k-2}$.

Next we consider the expression

$$
Q=(z n)_{k}-n_{k} z^{k}+n_{k}\left(k^{2}-k\right)\left(z^{k-1}-z^{k}\right) n^{-1} / 2 .
$$

Since the terms in $n^{k}$ and $n^{k-1}$ cancel out, we may apply our lemma to estimate each of the three terms of $Q$. Remembering $z \leqq 1$, we find $|Q|<C k^{7} n^{k-2}$ where $C$ depends only on $z$. We next note that $n^{m}(n-m)^{-m} \rightarrow 1$ as $n \rightarrow \infty$, so that $n^{k}(n-k)^{-k}$ has an upper bound independent of $n$ and $k$. Hence

$$
\left|Q / n_{k}\right|<C k^{7} n^{-2} n^{k}(n-k)^{-k}<C_{1} k^{7} n^{-2},
$$


and finally

$$
\sum_{0}^{m-1}(-a)^{k} Q / k ! n_{k}=O\left(n^{-2}\right) .
$$

If we substitute in (12) the expression for $Q$ given by (11) we verify that $S_{1}$ equals the right member of (9). Together with (10) this proves (9).

The same method, with more elaborate calculation, gives the general result (here again $\psi_{k}$ is defined in (4), and $z n$ is a positive integer not exceeding $n$ )

$$
\begin{aligned}
\left(1-a E+b E^{2} \cdots\right)^{2 n} \psi_{0}=e^{-a z}(a z)^{r}(r !)^{-1} \\
\cdot\left[1-\left(a^{2}+2 b-a^{2} z\right)\left(r^{2}-r-2 a r z+a^{2} z^{2}\right)\left(2 a^{2} z n\right)^{-1}+O\left(n^{-2}\right)\right],
\end{aligned}
$$

showing that the distribution of the number of "hits" obtained in the card-matching problems approaches the Poisson distribution with parameter $a z$. One may of course compute as many more terms of the asymptotic expansion as desired. (The author has computed the next two terms, but as the result is complicated and unverified, it will not be stated.)

For example, with a deck of $n$ cards divided into 4 suits, we found the probability of exactly $r$ matches to be

$$
\begin{aligned}
& \left(1-16 E+72 E^{2} \cdots\right)^{n / 4} \psi_{0} \\
& =e^{-4} 4^{r}(r !)^{-1}\left[1-21\left(r^{2}-9 r+16\right) / 8 n\right]+O\left(n^{-2}\right) .
\end{aligned}
$$

The probability of no matches $(r=0)$ is

$$
\left(1-16 E+72 E^{2} \cdots\right)^{n / 4} \phi_{0}=e^{-4}\left(1-42 n^{-1}\right)+O\left(n^{-2}\right) .
$$

However the approximation is still too crude for $n$ as small as $\mathbf{5 2}$.

5. The "n-kings problem." There is a second type of restricted permutations to which our method can be applied. Let it be required to find the probability that, in a permutation of $1, \cdots, n$, the integer $i$ does not immediately precede $j, k$ does not immediately precede $l$, and so on. We shall denote the probability that $i$ immediately precedes $j$ by $q_{i j}$. The expression to be evaluated is then

$$
\left(1-q_{i j}\right)\left(1-q_{k l}\right) \cdots \text {. }
$$

The fundamental requirement of quasi-symmetry (\$2) is fulfilled; in fact, the product of $k$ of the $q$ 's is either 0 or the same expression as in (4): $\phi_{k}=(n-k) ! / n !$. Moreover it is again true that $q_{i j} q_{k l}=0$ if $i=k$ or $j=l$; this time however we have the further complication $q_{i j} q_{j i}=0$, 
$q_{i j} q_{j k} q_{k i}=0$, and so on. With this modification, the calculations proceed as before.

The simplest problem of this kind requires merely that $i$ shall never precede $i+1$. We find

$$
\left(1-q_{12}\right)\left(1-q_{23}\right) \cdots\left(1-q_{n-1, n}\right)=(1-E)^{n-1} .
$$

A modification that suggests itself is to require further that $i+1$ shall not precede $i$. Or we can reformulate the question as a chess-board problem : in how many ways can $n$ kings be placed on an $n$ by $n$ board, one on each row and column, so that no two attack each other? We recognize a first cousin (albeit a shabby relative) of the famous " $n$ queens problem."

We have to evaluate

$$
A_{n}=\left(1-q_{12}\right)\left(1-q_{21}\right)\left(1-q_{28}\right)\left(1-q_{32}\right) \cdots\left(1-q_{n-1, n}\right)\left(1-q_{n, n-1}\right) \text {. }
$$

Denote by $B_{n}$ the result of deleting from $A_{n}$ the last factor. Then

$$
\begin{aligned}
& A_{n}=B_{n}\left(1-q_{n, n-1}\right), \\
& B_{n}=A_{n-1}\left(1-q_{n-1, n}\right) .
\end{aligned}
$$

Further we find

$$
A_{n-1} q_{n-1, n}=B_{n-1}\left(1-q_{n-1, n-2}\right) q_{n-1, n}=B_{n-1} q_{n-1, n} .
$$

From (14) and (15):

$$
B_{n}=A_{n-1}-B_{n-1} q_{n-1, n}
$$

On substituting (16) in (13) we get

$$
A_{n}=B_{n}-A_{n-1} q_{n, n-1} \text {. }
$$

Now in the product $B_{n-1} q_{n-1, n}$ it is readily checked that the latter factor does not enter into any vanishing products. Its effect therefore is simply to multiply the polynomial $B_{n-1}$ by $E$. That is to say, $B_{n-1} q_{n-1, n}=E B_{n-1}$, whence we can rewrite (16) as

$$
B_{n}=A_{n-1}-E B_{n-1} \text {. }
$$

Moreover it is clear from symmetry that $A_{n-1} q_{n-1, n}=A_{n-1} q_{n, n-1}$. From (15) and (17) we therefore obtain

$$
A_{n}=B_{n}-E B_{n-1} \text {. }
$$

Elimination of $A$ from (18) and (19) yields

'If there were a chess piece "*" which combined the moves of rook and king, the name " $n$-* problem" would be more suitable than $n$-kings. It might be added that the author has failed in several efforts to solve the $n$-queens problem by these methods. 


$$
B_{n}=(1-E) B_{n-1}-E B_{n-2} \text {. }
$$

The solution of the difference equation (20), with initial values $B_{1}=1$, $B_{2}=1-E$, is

$$
B_{n}=\left(u^{n}-v^{n}\right) /(u-v)
$$

where $u, v=\left(1-E \pm\left(1-6 E+E^{2}\right)^{1 / 2}\right) / 2 . A_{n}$ can be found from (19), and $A_{n} \phi_{0}$ is the solution of the $n$-kings problem. The first 3 terms are:

$$
A_{n}=1-(2 n-2) E+\left(2 n^{2}-8 n+8\right) E^{2} \cdots .
$$

Check for $n=4$ :

$$
\begin{aligned}
B_{4} & =u^{3}+u^{2} v+u v^{2}+v^{3}=1-5 E+5 E^{2}-E^{3}, \\
B_{3} & =u^{2}+u v+v^{2}=1-3 E+E^{2}, \\
A_{4} & =B_{4}-E B_{3}=1-6 E+8 E^{2}-2 E^{3}, \\
A_{4} \phi_{0} & =(4 !-6 \cdot 3 !+8 \cdot 2 !-2 \cdot 1 !) / 4 !=2 / 4 !,
\end{aligned}
$$

corresponding to the two permissible arrangements 3142 and 2413.

6. A problem of Netto. In [7, p. 84], the following problem is proposed. Let the integers $1, \cdots, n$ be divided into $n / a$ sets of $a$ each. What is the probability that in a permutation there are nowhere two consecutive elements from the same subset? Thus for a deck of cards $(a=4)$, we require the probability of going through the deck and never encountering two consecutive cards of the same denomination (aces, deuces, and so on).

If the integers $1, \cdots, a$ are taken to form the first subset, we have to compute

$$
V_{a}=\prod\left(1-q_{i j}\right)
$$

the product taken over all $i, j \leqq a$ with $i \neq j$; then the answer to Netto's problem will be $V_{a}^{n / a}$. Let $W_{a}$ denote the result of deleting from $V_{a}$ the $a-1$ factors $\left(1-q_{i a}\right)(i=1, \cdots, a)$. Then

$$
V_{a}=W_{a}\left(1-q_{1 a}\right) \cdots\left(1-q_{a-1, a}\right)=W_{a}-(a-1) W_{a} q_{a-1, a} .
$$

Now in the product $W_{a} q_{a-1, a}$ the effect of multiplication by $q_{a-1, a}$ will be to delete from $W_{a}$ the remaining terms of the form $q_{a-1, i}$ and also the term $q_{a, a-1}$. In the product that remains it is now merely a harmless change of notation to replace $a$ by $a-1$, after which precisely $V_{a-1}$ is left. Hence

$$
\begin{gathered}
W_{a} q_{a-1, a}=E V_{a-1}, \\
V_{a}=W_{a}-(a-1) E V_{a-1} .
\end{gathered}
$$


By a repetition of virtually the same argument we can show that

$$
W_{a}=V_{a-1}-(a-1) E W_{a-1} .
$$

By eliminating $W$ from (21) and (22), we obtain

$$
V_{a}=(1+2 E-2 a E) V_{a-1}-(a-1)(a-2) E^{2} V_{a-2} \text {. }
$$

The solution of this difference equation with initial values $V_{1}=1$, $V_{2}=1-2 E$ is $F(a, a-1)$ in the notation of (5), Our final answer is thus $[F(a, a-1)]^{n / a} \phi_{0}$.

With $a=4$ the result becomes

$$
\left(1-12 E+36 E^{2}-24 E^{3}\right)^{n / 4} \phi_{0}=e^{-3}+O\left(n^{-1}\right) .
$$

The odds are about 19 to 1 , although to the author's knowledge this game has been played at even money!

\section{BIBLIOGRAPHY}

1. I. L. Battin, On the problem of multiple matching, Annals of Mathematical Statistics vol. 13 (1942) pp. 294-305.

2. T. S. Broderick, On some symbolic formulae in probability theory, Proceedings of the Royal Irish Academy vol. 44 (1937) pp. 19-28.

3. M. Fréchet, Note on the problème des rencontres, Amer. Math. Monthly vol. 46 (1939) p. 501.

4. I. Kaplansky, On a generalization of the probleme des rencontres, Amer. Math. Monthly vol. 46 (1939) pp. 159-161.

5. - Solution of the probleme des mènages, Bull. Amer. Math. Soc. vol. 49 (1943) pp. 784-785.

6. E. Lucas, Theorie des nombres, Paris, 1891.

7. E. Netto, Lehrbuch der Combinatorik, Berlin, 1927.

8. J. Touchard, Sur un probleme de permutations, C. R. Acad. Sci. Paris vol. 198 (1934) pp. 631-633.

HaRvard UNIVERSITY 